\title{
Evapotranspiração de referência estimada por modelos simplificados para o Estado do Mato Grosso
}

\author{
Adriana Aki Tanaka(1), Adilson Pacheco de Souza(1), Antonio Evaldo Klar(2), \\ Andréa Carvalho da Silva ${ }^{(1)}$ e Anthony Wellington Almeida Gomes ${ }^{(3)}$
}

\begin{abstract}
(1)Universidade Federal de Mato Grosso, Campus Universitário de Sinop, Instituto de Ciências Agrárias e Ambientais, Avenida Alexandre Ferronato, oㅜ 1.200, CEP 78557-267 Sinop, MT, Brasil. E-mail: dritanak@hotmail.com, pachecoufmt@gmail.com, acarvalho@ufmt.br (2)Universidade Estadual Paulista Júlio de Mesquita, Campus de Botucatu, Faculdade de Ciências Agronômicas, Departamento de Engenharia Rural, Fazenda Lageado, Rua José Barbosa de Barros, № 1.780, CEP 18610-307 Botucatu, SP, Brasil. E-mail: klar@fca.unesp.br (3)Universidade Federal Rural do Pernambuco, Unidade Acadêmica de Garanhuns, Avenida Bom Pastor, s/no, Boa Vista, CEP 55292-270 Garanhuns, PE, Brasil. E-mail: awagomes@uag.ufrpe.br
\end{abstract}

Resumo - O objetivo deste trabalho foi avaliar o desempenho de 12 modelos simplificados de estimativa da evapotranspiração de referência (ETo), para o Estado do Mato Grosso. Os dados foram obtidos da rede de estações meteorológicas automáticas (EMAs) do Instituto Nacional de Meteorologia, localizadas em 28 municípios do Estado. Os modelos simplificados de estimativa avaliados foram os de: Hargreaves-Samani, Camargo, Makkink, Linacre, McGinness-Bordne, Romanenko, Turc, Holdridge, Radiação Solar, Jensen-Haise, Hansen e Caprio. Adotou-se o método de Penman-Monteith Fao 56 (PMF) como referência na avaliação das estimativas simplificadas. O desempenho estatístico foi avaliado por meio do erro absoluto médio (MBE), da raiz quadrada do erro quadrático médio (RMSE), do índice de concordância (d) de Willmott e de acordo com o ordenamento numérico dos modelos para cada índice. O modelo de Makkink superestimou a ETo de 2,0 a 3,0 mm por dia, com espalhamentos de $2,75 \mathrm{~mm}$ por dia e índice d de 0,40 , o que resultou nos piores desempenhos entre os modelos, independentemente do município avaliado. Os modelos de Turc e McGinness-Bordne apresentaram os melhores desempenhos de estimativa da ETo, para 57,1 e 25\% das EMAs, respectivamente. Os modelos de Romanenko, Makkink e Holdridge não são recomendados para o Estado do Mato Grosso.

Termos para indexação: dados mínimos, modelo de Turc, Penman-Monteith, radiação solar, temperatura do ar.

\section{Reference evapotranspiration estimated with simplified models for the state of Mato Grosso, Brazil}

\begin{abstract}
The objective of this work was to evaluate the performance of 12 simplified models for the estimation of reference evapotranspiration (ETo) for the state of Mato Grosso, Brazil. The data were collected from automatic weather stations (AWS) of the Instituto Nacional de Meteorologia, located in 28 municipalities of the state. The following simplified estimation models were evaluated: Hargreaves-Samani, Camargo, Makkink, Linacre, McGinness-Bordne, Romanenko, Turc, Holdridge, Solar Radiation, Jensen-Haise, Hansen, and Caprio. The Fao 56 Penman-Monteith method (FPM) was used as reference for assessing the simplified estimates. Statistical performance was evaluated through relative mean error (RME), root mean square error (RMSE), Willmott's d index, and according to the numerical order of models for each index. The Makkink model overestimated ETo by 2.0 to $3.0 \mathrm{~mm}$ per day, with scattering values of $2.75 \mathrm{~mm}$ per day and $0.40 \mathrm{~d}$ index, which represented the worst results among models, regardless of the municipality evaluated. The Turc and McGinness-Bordne models showed the best performances for estimating ETo in 57.1 and $25 \%$ of the AWS, respectively. The Romanenko, Makkink, and Holdridge models are not recommended for the state of Mato Grosso, Brazil.
\end{abstract}

Index terms: minimum data, Turc model, Penman-Monteith, solar radiation, air temperature.

\section{Introdução}

O crescimento populacional, a demanda por alimentos e os níveis de industrialização têm acarretado aumento do consumo dos recursos hídricos em escalas regionais e locais, o que exige um planejamento eficiente de sua oferta e demanda, para diferentes escalas temporais. Entre os diferentes usos desses recursos, a irrigação é uma das atividades com maior consumo de água (Bezerra et al., 2010). 
A evapotranspiração (ET) é o principal componente no balanço de água em ecossistemas agrícolas, além de ser um fator-chave nos estudos de avaliação ambiental, no manejo de bacias hidrográficas e na estimativa da necessidade hídrica das culturas (Raziei \& Pereira, 2013; Carvalho et al., 2015). De maneira simplificada, a ET pode ser definida como um processo simultâneo e dinâmico de mudança do estado físico da água pela evaporação no solo e pela transpiração das plantas (Alencar et al., 2011). A evapotranspiração de referência (ETo) indica a perda de água de uma superfície vegetada, em crescimento ativo e sem restrição hídrica, que cobre totalmente o solo (Pereira et al., 1997; Allen et al., 1998).

A ET é um processo dependente da disponibilidade energética, da demanda atmosférica, da disponibilidade de água do solo e de fatores fisiológicos da planta (Souza et al., 2011). Já a ETo pode ser obtida por meio de técnicas diretas, com a utilização de lisímetros; ou por métodos indiretos, por meio de modelos físico-matemáticos de estimativa (Alves Sobrinho et al., 2011).

Segundo Henrique \& Dantas (2007), os métodos empíricos de estimativa da ETo apresentam muitas limitações. Entre estas, a ausência de informações que permitam a utilização dos métodos mais consistentes tem estimulado o desenvolvimento de métodos simplificados para a determinação da ETo. Além disso, a estimativa do consumo de água pelas plantas sem critérios técnicos, ou com base em modelos com baixo desempenho, tem resultado em super ou subdimensionamento de projetos de irrigação ou em manejo inadequado da água (Fernandes et al., 2011; Carvalho et al., 2015).

Os modelos simplificados procuram estimar a ETo de maneira acurada, com o emprego de menor número possível de variáveis (Carvalho et al., 2011). Atualmente, a equação combinada de Penman-Monteith, parametrizada pela FAO no Boletim 56 (Allen et al., 1998), apresenta as melhores representações dos fatores físicos e fisiológicos que regem a ETo (Bezerra et al., 2010b; Souza et al., 2011; Carvalho et al., 2015). No entanto, a sua aplicação ainda exige grande número de parâmetros, que geralmente não estão disponíveis nas estações meteorológicas, por ausência de sensores ou pela baixa qualidade dos dados.

Para minimizar esses problemas, calibrações locais e regionais, aliadas a avaliações de desempenho estatístico dos modelos, são importantes para avaliar os erros nas estimativas da ETo e nortear a escolha dos métodos que melhor se adequem à disponibilidade local de dados meteorológicos e ao nível de precisão exigido (Tagliaferre et al., 2010; Carvalho et al., 2011; Souza et al., 2011; Carvalho et al., 2015). Neste sentido, a literatura não apresenta registros desse tipo de informação para o Estado do Mato Grosso que, entre os estados brasileiros, destaca-se por sua grande extensão territorial. $O$ Estado se insere na área de ocorrência do Cerrado brasileiro, da Floresta Tropical Úmida e da planície do Pantanal, o que indica ocorrência de cenários ambientais bastante distintos, especialmente quanto às variáveis climáticas.

O objetivo deste trabalho foi avaliar o desempenho de 12 modelos simplificados de estimativa da evapotranspiração de referência (ETo), para o Estado do Mato Grosso.

\section{Material e Métodos}

Os dados foram obtidos da rede de estações automáticas do Instituto Nacional de Meteorologia (Inmet), localizadas em 28 municípios do Mato Grosso (Figura 1), com intervalos de coleta diferenciados em função da época de instalação ou de funcionamento das estações (Tabela 1). Apesar de as séries históricas terem sido pequenas, a indisponibilidade de informações para estudos dessa natureza tem feito com que diversas pesquisas sejam desenvolvidas com séries ainda menores (Carvalho et al., 2015). Os dados horários de temperatura do ar e umidade relativa do ar (média, máxima e mínima) de cada estação foram analisados para obtenção dos valores médios diários de todos os elementos meteorológicos.

No Estado do Mato Grosso, há duas estações climáticas bem definidas: a chuvosa, de outubro a abril, e a seca, de maio a setembro, com precipitações totais entre 1.200 e $2.000 \mathrm{~mm}$. As maiores precipitações ocorrem nas regiões Norte do Estado e nas regiões com altitudes próximas a $800 \mathrm{~m}$, com menores níveis nas direções leste, oeste e sul, e uma distribuição espacial e temporal irregular.

Pela classificação climática de Köppen, predominam os tipos climáticos Aw (clima tropical) e Cwa (clima temperado ou tropical de altitude), com chuvas concentradas no verão/outono e deficiências hídricas no inverno/primavera, sendo que as regiões Norte, Sul, Sudeste e Serrana são as mais úmidas, quando 
comparadas à Baixada Cuiabana e ao Pantanal (Souza et al., 2013).

A ETo utilizada para avaliação dos modelos simplificados foi obtida com o método de Penman-Monteith (PMF), parametrizado pelo Boletim FAO 56 (Allen et al., 1998). Em geral, nas estações automáticas da rede do Inmet, as variáveis temperatura e umidade do ar são monitoradas a $2,0 \mathrm{~m}$ de altura, e a velocidade do vento, a $10 \mathrm{~m}$ de altura, com uso de anemômetros de canecas. Para a aplicação do PMF, as demais variáveis foram obtidas de acordo com as recomendações de Pereira et al. (1997) e Allen et al. (1998). O método é descrito pela seguinte equação:

$$
\mathrm{ET}_{\mathrm{o}}=\frac{0,408 \Delta(\mathrm{Rn}-\mathrm{G})+\gamma \frac{900}{\mathrm{~T}_{\text {méd }}+278} \mathrm{u}_{2}\left(\mathrm{e}_{\mathrm{s}}-\mathrm{e}_{\mathrm{a}}\right)}{\Delta+\gamma\left(1+0,34 \mathrm{u}_{2}\right)}
$$

em que: $\mathrm{ET}_{\mathrm{o}}$ é a evapotranspiração de referência (mm por dia); Rn é a radiação líquida ( $\mathrm{MJ} \mathrm{m}^{-2}$ por dia); $\mathrm{G}$ é o fluxo de calor no solo ( $\mathrm{MJ} \mathrm{m}^{-2}$ por dia); $\mathrm{T}_{\text {méd }}$ é a

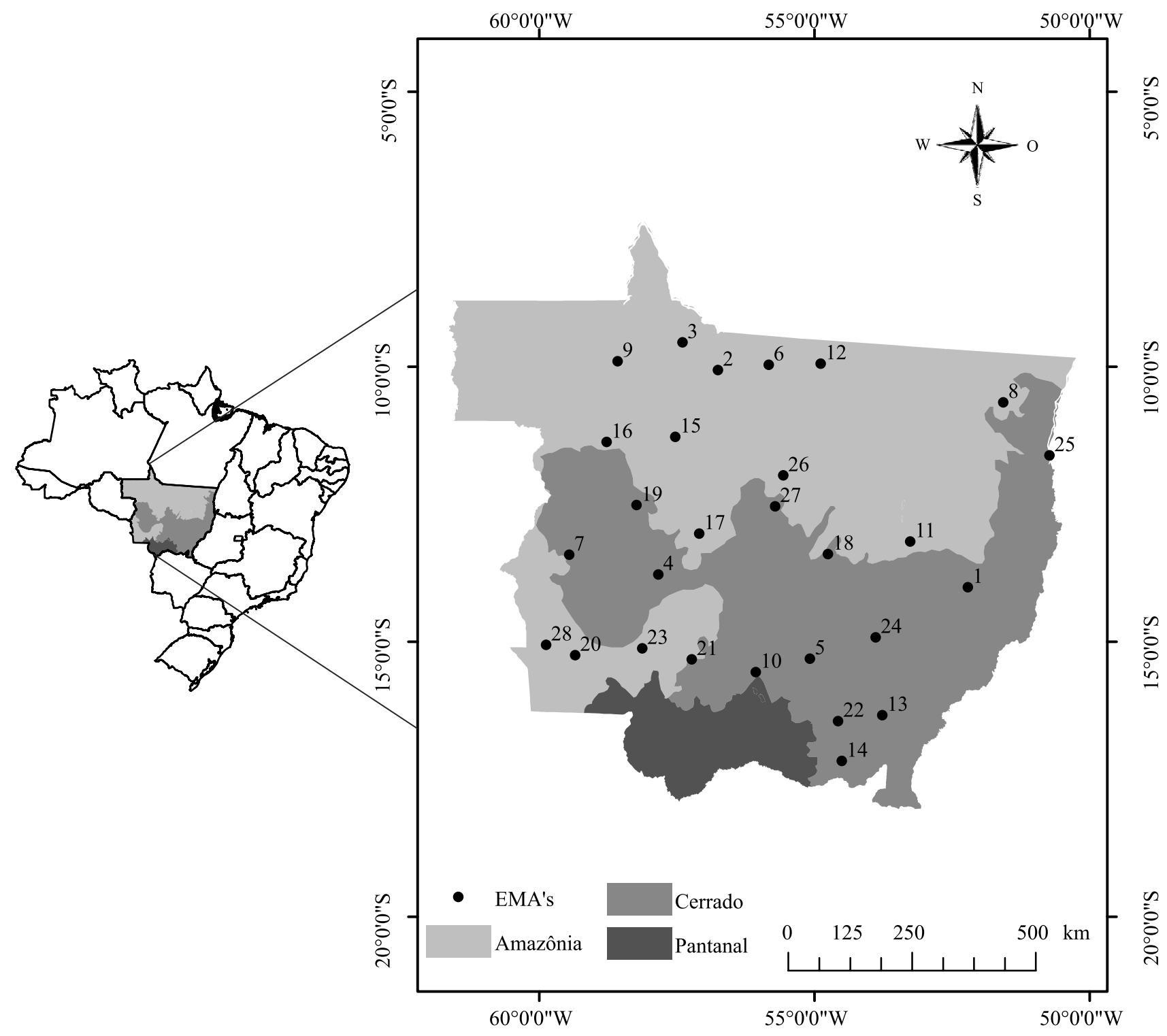

Figura 1. Localização das estações meteorológicas automáticas estudadas e dos biomas presentes no Estado do Mato Grosso. Fonte: adaptado de Secretaria de Meio Ambiente do Estado do Mato Grosso, SEMA/MT (2013). 
média diária da temperatura do ar a $10,0 \mathrm{~m}\left({ }^{\circ} \mathrm{C}\right) ; \mathrm{u}_{2}$ é a

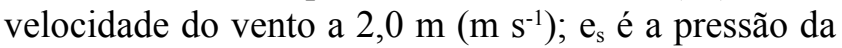
saturação de vapor $(\mathrm{kPa})$; $\mathrm{e}_{\mathrm{a}}$ é a pressão de vapor atual $(\mathrm{kPa}) ;\left(\mathrm{e}_{\mathrm{s}}-\mathrm{e}_{\mathrm{a}}\right)$ é o deficit de saturação de vapor $(\mathrm{kPa})$; $\Delta$ é a declividade da curva da pressão de vapor vs. temperatura $\left(\mathrm{kPa}^{\circ} \mathrm{C}^{-1}\right)$; e $\gamma$ é a constante psicrométrica $\left(\mathrm{kPa}^{\circ} \mathrm{C}^{-1}\right)$. A constante 0,408 corresponde a $1 / \lambda$, em que $\lambda$ é o calor latente de vaporização da água $\left(2,45 \mathrm{MJ} \mathrm{kg}^{-1}\right)$; e a constante 900 , a um fator de conversão referente à escala diária de dados.

Para o cenário de dados meteorológicos limitados, foram empregados apenas dados de temperatura do ar e umidade relativa do ar, e, quando necessário, foi estimada a radiação solar global pelo modelo de Bristow \& Campbell (1984), com coeficientes regionalizados por Tanaka (2013). Nesse contexto, foram avaliados 12 modelos de estimativa da ETo diária, como segue.

O modelo de Hargreaves-Samani segundo Carvalho et al., (2015) é definido pela expressão:
$\mathrm{ET}_{\mathrm{o}}=0,0023 \mathrm{H}_{\mathrm{o}}\left(\mathrm{T}_{\text {máx }}-\mathrm{T}_{\text {min }}\right)^{0,5}\left(\mathrm{~T}_{\text {méd }}+17,8\right)$, em que $\mathrm{H}_{0}$ é a radiação extraterrestre em equivalente de evaporação (mm por dia); e $\mathrm{T}_{\text {máx }}, \mathrm{T}_{\text {mín }}$ e $\mathrm{T}_{\text {méd }}$ são as temperaturas $\left({ }^{\circ} \mathrm{C}\right)$ máxima, mínima e média diária, respectivamente.

Já o modelo de Camargo (Pereira et al., 1997) é definido pela expressão: $\mathrm{ET}_{\mathrm{o}}=\mathrm{F} \times \mathrm{H}_{\mathrm{o}} \times \mathrm{T}_{\text {med }} \times \mathrm{ND}$, em que $\mathrm{F}$ é um fator de ajuste que varia de acordo com a temperatura média anual do local $-\mathrm{F}=0,01$ para $\mathrm{T}$ até $23^{\circ} \mathrm{C}, \mathrm{F}=0,0105$ para $\mathrm{T}=24^{\circ} \mathrm{C}, \mathrm{F}=0,011$ para $\mathrm{T}=25^{\circ} \mathrm{C}, \mathrm{F}=0,0115$ para $\mathrm{T}=26^{\circ} \mathrm{C}$ e $\mathrm{F}=0,012$ para $\mathrm{T}>26^{\circ} \mathrm{C}$; e ND é o número de dias do período.

O modelo de Makkink (Pereira et al., 1997) é definido pela expressão: $\mathrm{ET}_{\mathrm{o}}=0,61 \times \mathrm{W} \times \mathrm{H}_{\mathrm{G}}-0,12$, em que $\mathrm{H}_{\mathrm{G}}$ é a radiação solar global diária, em equivalente de evaporação (mm por dia); e W é um fator de ponderação, que pode ser calculado para as condições $\mathrm{W}=0,407+0,01475 \mathrm{~T}$, quando $0<\mathrm{T}<16^{\circ} \mathrm{C}$, ou $\mathrm{W}=$ $0,483+0,01 \mathrm{~T}$, quando $16,1<\mathrm{T}<32^{\circ} \mathrm{C}$ (Viswanadham et al., 1991).

Tabela 1. Identificação das estações meteorológicas automáticas avaliadas no Estado do Mato Grosso.

\begin{tabular}{|c|c|c|c|c|c|c|c|c|}
\hline Código & $\begin{array}{c}\text { Nome } \\
\text { da estação }\end{array}$ & $\begin{array}{l}\text { Latitude } \\
\text { (S) }\end{array}$ & $\begin{array}{l}\text { Longitude } \\
\text { (W) }\end{array}$ & $\begin{array}{l}\text { Altitude } \\
(\mathrm{m})\end{array}$ & $\begin{array}{l}\text { Período } \\
\text { (mês/ano) }\end{array}$ & $\begin{array}{l}\text { Número } \\
\text { de dados }\end{array}$ & $\begin{array}{c}\text { Dados } \\
\text { efetivos }\end{array}$ & $\begin{array}{c}\text { Perdas } \\
(\%)\end{array}$ \\
\hline A-908 & 1. Água Boa & $-14,0161$ & $-52,2122$ & 432 & $1 / 2008-1 / 2013$ & 1.858 & 1.631 & 12,22 \\
\hline A-924 & 2. Alta Floresta & $-10,0672$ & $-56,7522$ & 294 & $9 / 2011-1 / 2013$ & 519 & 422 & 18,69 \\
\hline A-910 & 3. Apiacás & $-9,5639$ & $-57,3936$ & 220 & $10 / 2006-1 / 2013$ & 2.315 & 1.364 & 41,08 \\
\hline A-905 & 4. Campo Novo dos Parecis & $-13,7833$ & $-57,8333$ & 570 & $6 / 2010-1 / 2013$ & 976 & 505 & 48,26 \\
\hline A-912 & 5. Campo Verde & $-15,3139$ & $-55,0808$ & 749 & $1 / 2008-1 / 2013$ & 1.858 & 898 & 51,67 \\
\hline A-926 & 6. Carlinda & $-9,9703$ & $-55,8272$ & 300 & $4 / 2008-1 / 2013$ & 1.768 & 1.517 & 14,20 \\
\hline A-913 & 7. Comodoro & $-13,4231$ & $-59,4546$ & 591 & $1 / 2008-1 / 2013$ & 1.858 & 1.511 & 18,68 \\
\hline A-918 & 8. Confresa & $-10,6539$ & $-51,5668$ & 237 & $6 / 2008-1 / 2013$ & 1.707 & 1.278 & 25,13 \\
\hline A-919 & 9. Cotriguaçu & $-9,9061$ & $-58,5719$ & 261 & $1 / 2008-1 / 2013$ & 1.858 & 1.564 & 15,82 \\
\hline A-901 & 10. Cuiabá & $-15,5594$ & $-56,0628$ & 240 & $5 / 2011-1 / 2013$ & 642 & 463 & 27,88 \\
\hline A-930 & 11. Gaúcha do Norte & $-13,1847$ & $-53,2575$ & 379 & $8 / 2008-1 / 2013$ & 1.646 & 1.376 & 16,40 \\
\hline A-906 & 12. Guarantã & $-9,9500$ & $-54,8833$ & 320 & $5 / 2007-1 / 2013$ & 2.102 & 1.338 & 36,35 \\
\hline A-932 & 13. Guiratinga & $-16,3417$ & $-53,7661$ & 526 & $1 / 2008-1 / 2013$ & 1.858 & 1.201 & 35,36 \\
\hline A-933 & 14. Itiquira & $-17,1750$ & $-54,5014$ & 585 & $8 / 2008-1 / 2013$ & 1.646 & 981 & 40,40 \\
\hline A-914 & 15. Juara & $-11,2803$ & $-57,5267$ & 260 & $11 / 2006-2 / 2012$ & 1.947 & 1.265 & 35,03 \\
\hline A-920 & 16. Juína & $-11,3750$ & $-58,7750$ & 374 & $10 / 2007-1 / 2013$ & 1.949 & 1.259 & 35,40 \\
\hline A-928 & 17. Nova Maringá & $-13,0386$ & $-57,0922$ & 353 & $4 / 2008-1 / 2013$ & 1.768 & 975 & 44,85 \\
\hline A-929 & 18. Nova Ubiratã & $-13,4111$ & $-54,7522$ & 518 & $4 / 2008-1 / 2013$ & 1.768 & 1.168 & 33,94 \\
\hline A-927 & 19. Novo Mundo & $-12,5219$ & $-58,2314$ & 431 & $3 / 2008-1 / 2013$ & 1.798 & 1.373 & 23,64 \\
\hline A-917 & 20. Pontes de Lacerda & $-15,2511$ & $-59,3467$ & 256 & $1 / 2008-1 / 2013$ & 1.858 & 1.301 & 29,98 \\
\hline A-935 & 21. Porto Estrela & $-15,3247$ & $-57,2264$ & 145 & $2 / 2008-1 / 2013$ & 1.827 & 767 & 58,02 \\
\hline A-907 & 22. Rondonópolis & $-16,4500$ & $-54,5666$ & 284 & $1 / 2008-1 / 2013$ & 1.858 & 1.377 & 25,89 \\
\hline A-936 & 23. Salto do Céu & $-15,1247$ & $-58,1275$ & 303 & $1 / 2008-1 / 2013$ & 1.858 & 1.462 & 21,31 \\
\hline A-931 & 24. Santo Antônio do Leste & $-14,9278$ & $-53,8836$ & 648 & $8 / 2008-1 / 2013$ & 1.646 & 1.238 & 24,79 \\
\hline A-921 & 25. São Félix do Araguaia & $-11,6189$ & $-50,7278$ & 218 & $8 / 2011-1 / 2013$ & 550 & 456 & 17,09 \\
\hline A-917 & 26. Sinop & $-11,9822$ & $-55,5658$ & 371 & $11 / 2006-6 / 2012$ & 2.284 & 930 & 59,28 \\
\hline A-904 & 27. Sorriso & $-12,5452$ & $-55,7113$ & 380 & $1 / 2009-1 / 2013$ & 1.493 & 958 & 35,83 \\
\hline A-922 & 28. Vila Bela da Santíssima Trindade & $-15,0628$ & $-59,8729$ & 222 & $1 / 2008-1 / 2013$ & 1.858 & 1.404 & 24,43 \\
\hline
\end{tabular}


O modelo de Linacre (Pereira et al., 1997) é definido pela equação:

$$
\mathrm{ET}_{0}=\frac{\frac{\mathrm{J}\left(\mathrm{T}_{\text {méd }}+0,006 \mathrm{~h}\right)}{100-\varphi}+15\left(\mathrm{~T}_{\text {méd }}-\mathrm{T}_{\mathrm{PO}}\right)}{80-\mathrm{T}_{\text {méd }}}
$$

em que: J é a constante igual a 500, no caso de vegetação $(\mathrm{r}=0,25)$, e a 700 , no caso de água livre; $\mathrm{h}$ é a altitude do local em metros; ø é a latitude local em graus (em módulo); e $\mathrm{T}_{\mathrm{PO}}$ é a temperatura média diária do ponto de orvalho $\left({ }^{\circ} \mathrm{C}\right)$, sendo que $\left(\mathrm{T}_{\text {méd }}-\mathrm{T}_{\mathrm{PO}}\right)=0,0023 \mathrm{~h}+$ $0,37 \mathrm{~T}_{\text {méd }}+0,53\left(\mathrm{~T}_{\text {máx }}-\mathrm{T}_{\text {mín }}\right)+0,35 \mathrm{R}-10,9$; neste caso, $R$ é a diferença entre a temperatura média dos meses mais quentes e frios.

O modelo de McGinness-Bordne (Oudin et al., 2005), por sua vez, é definido pela equação:

$$
\mathrm{ET}_{0}=\left(\frac{\mathrm{H}_{0}}{\lambda \rho}\right)\left[\frac{\mathrm{T}_{\text {méd }}+5}{68}\right]
$$

em que: $\mathrm{H}_{0}$ é a radiação extraterrestre ( $\mathrm{MJ} \mathrm{m}^{-2}$ por dia); $\lambda$ é o calor latente de vaporização $\left(\mathrm{MJ} \mathrm{kg}^{-1}\right)$; e $\rho$ é a densidade da água $\left(\mathrm{kg} \mathrm{m}^{-3}\right)$.

O modelo de Romanenko (Oudin et al., 2005) é definido pela equação:

$$
\mathrm{ET}_{0}=4,5\left[1+\frac{\mathrm{T}_{\text {méd }}}{25}\right]^{2}\left(1-\frac{\mathrm{e}_{\mathrm{a}}}{\mathrm{e}_{\mathrm{s}}}\right)
$$

em que: $\mathrm{e}_{\mathrm{a}}$ é a pressão atual de vapor $(\mathrm{kPa})$; e $\mathrm{e}_{\mathrm{s}}$ é a pressão média de saturação de vapor $(\mathrm{kPa})$.

O modelo de Turc (Carvalho et al., 2015) é definido pelas expressões:

$$
\mathrm{ET}_{0}=0,013\left[\frac{\mathrm{T}_{\text {méd }}}{\mathrm{T}_{\text {méd }}+15}\right]\left(23,8846 \mathrm{H}_{0}+50\right)\left(1+\frac{50-\mathrm{UR}}{70}\right)
$$

para $\mathrm{UR}<50 \% ; \quad \mathrm{e} \quad \mathrm{ET}_{\mathrm{o}}=0,013\left[\mathrm{~T}_{\text {méd }} / \mathrm{T}_{\text {méd }}+15\right]$ $\left(23,8846 \mathrm{H}_{\mathrm{o}}+50\right)$, para UR $>50 \%$, em que $\mathrm{H}_{0}$ é a radiação extraterrestre diária $\left(\mathrm{MJ} \mathrm{m}^{-2}\right.$ por dia).

O modelo de Holdridge (Holdridge, 1959) é definido pela equação: $\mathrm{ET}_{\mathrm{o}}=[58,93(\mathrm{Put} / \mathrm{Nut})] \mathrm{T}_{\text {méd, }}$, em que: Put é o período por unidade de tempo (dias); e Nut é o número de unidades de tempo em um ano (365 ou 366 dias).

O modelo de Radiação Solar (Pereira et al., 1997) é definido pela expressão $\mathrm{ET}_{\mathrm{o}}=\mathrm{c} \times \mathrm{W} \times \mathrm{Hg}$, em que $\mathrm{W}$ é obtido pelo método de Makkink; e c é o coeficiente que representa a inclinação da reta de regressão, obtido como descrito em Doorenbos \& Kassan (1994) e Pereira et al. (1997).

O modelo Jensen-Haise (Pereira et al., 1997) é definido pela expressão $\mathrm{ET}_{\mathrm{o}}=\mathrm{H}_{\mathrm{G}}\left(0,00252 \mathrm{~T}_{\text {méd }}+0,078\right)$.

Já o modelo de Hansen (Hansen, 1984) é definido pela equação:

$$
\mathrm{ET}_{0}=0,7\left[\frac{\Delta}{\Delta+\gamma}\right]\left(\frac{\mathrm{H}_{\mathrm{G}}}{\lambda}\right)
$$

com variáveis definidas no PMF.

Por fim, o modelo de Caprio (Caprio, 1974) é definido pela equação:

$$
\mathrm{ET}_{0}=\left[\begin{array}{ll}
\frac{6,1}{10^{6}} & \mathrm{H}_{\mathrm{G}}\left(1,8 \mathrm{~T}_{\text {méd }}+1,0\right)
\end{array}\right]
$$

em que $\mathrm{H}_{\mathrm{G}}$ é a radiação solar global média diária (KJ $\mathrm{m}^{-2}$ por dia).

Para a avaliação do desempenho estatístico dos modelos, foram empregados os indicadores de erro absoluto médio (MBE), raiz quadrada do erro quadrático médio (RMSE) e índice de Willmott (d). Conforme Souza et al. (2011), a utilização conjunta desses indicadores estatísticos pode ser considerada como uma boa alternativa para validação de modelos estatísticos. Isso porque esses indicadores permitem a análise simultânea do desvio da média e a identificação da ocorrência de sub ou superestimativa, espalhamento e ajustamento dos modelos, quando comparados a valores estimados e medidos. Os indicadores foram calculados de acordo com as seguintes equações:

$$
\begin{gathered}
\text { MBE }=\frac{\sum_{i=1}^{N}\left(P_{i}-O_{i}\right)}{N-1} ; \operatorname{RMSE}=\left[\frac{\sum_{i=1}^{N}\left(P_{i}-O_{i}\right)^{2}}{N}\right]^{0,5} ; \\
d=\left[\frac{\sum_{i=1}^{N}\left(P_{i}-O_{i}\right)^{2}}{\sum\left(\left|P_{i}-O\right|+\left|O_{i}-O\right|\right)^{2}}\right] ;
\end{gathered}
$$

em que $\mathrm{P}_{\mathrm{i}}$ são os valores estimados pelos modelos simplificados (mm por dia); $\mathrm{O}_{\mathrm{i}}$ são os valores estimados pelo PMF (mm por dia); O é a média dos valores 
estimados pelo PMF (mm por dia); e N é o número de valores.

Em geral, o indicador MBE representa o desvio das médias e provê informações sobre o desempenho do modelo em longo prazo; valores negativos indicam subestimativa, e vice-versa. Quanto menor o valor absoluto de MBE, melhor é o desempenho do modelo testado (Stone, 1993); porém, uma superestimativa cancela uma subestimativa. Já o indicador estatístico RMSE informa sobre o valor real do erro produzido pelo modelo e indica o espalhamento das estimativas da variável independente, para um determinado valor da variável dependente. Contudo, erros de grande proporção na soma podem causar acréscimos significativos nos valores de RMSE, e, além disso, o indicador não diferencia superestimativas de subestimativas. Quanto menores os valores obtidos para RMSE, melhor o desempenho dos modelos. Por sua vez, o índice de ajustamento d de Willmott, que varia de 0 a 1 , representa o quanto os valores estimados ajustam-se a valores medidos, ou seja, é uma medida de quão bem o modelo estima o afastamento dos dados da média observada (Willmott, 1985).

Posteriormente, utilizaram-se os valores ponderados (Vp) dos indicadores estatísticos para classificar e definir o melhor método para a estimativa da ETo. Para a obtenção dos Vp, atribuíram-se pesos de 1 a $\mathrm{n}$ para cada indicador estatístico, em que n é o número de modelos testados que, nesse caso, tiveram peso 1 , para o modelo com melhor desempenho, e peso n, para o modelo com pior desempenho. O ordenamento dos modelos de acordo com seu desempenho foi feito por meio do somatório dos pesos atribuídos em cada estação; ou seja, o menor valor ponderado acumulado indicou o melhor modelo (Monteiro et al., 2014).

\section{Resultados e Discussão}

Em geral, observaram-se tendências de superestimativas dos valores de ETo pelos modelos simplificados (Tabela 2), com exceção dos modelos de McGinness-Bordne e Caprio. As estimativas da ETo obtidas pelos modelos de Hansen, Radiação Solar, McGinness-Bordne e Camargo apresentaram os menores valores de MBE para 8, 6, 5 e 4 EMAs, respectivamente. Já o modelo de Makkink apresentou superestimativa da ETo de 1,82 a 2,89 $\mathrm{mm}$ por dia, e o modelo de Caprio subestimou a ETo em 27 EMAs, em até $1,08 \mathrm{~mm}$ por dia. Esses comportamentos indicam que as estimativas da ETo melhoram quando os modelos que empregam a radiação solar global são utilizados no estado de Mato Grosso.

Os menores desvios para os valores médios foram obtidos pelos modelos de Hansen e Radiação Solar, que contemplam a radiação global, dada pelo modelo de Bristow \& Campbell (1984) - baseado apenas na temperatura do ar -, com uso dos coeficientes regionalizados por Tanaka (2013). Portanto, mesmo com erros nas estimativas da radiação global, melhores respostas são obtidas com esses modelos, em comparação aos que englobam a radiação extraterreste, isto é, os de Turc, McGinness-Bordne, Hargreaves-Samani e Camargo. Segundo Souza et al. (2011), o desempenho da estimativa da ETo para um mesmo modelo varia entre regiões úmidas, semiúmidas ou áridas, pois a sazonalidade da ETo é influenciada pela variação da quantidade de vapor d'água na atmosfera local, que, por sua vez, está relacionada à nebulosidade e sofre influência direta do balanço de radiação entre ondas curtas e longas, com reflexos sobre o balanço (Carvalho et al., 2015).

Esses resultados corroboram os de Conceição \& Mandelli (2005), que avaliaram os métodos de Hargreaves-Samani, Camargo, Thornthwaite, Thornthwaite modificado, Makkink e Radiação Solar para estimativa da ETo, em escala mensal, no município de Bento Gonçalves, RS. Os autores observaram que os modelos que usam a radiação solar incidente (Makkink e Radiação Solar) apresentam desempenho superior ao dos modelos que usam apenas a temperatura do ar. No entanto, Vescove \& Turco (2005), ao estudar os métodos de Radiação Solar, Makkink e Tanque classe A, para a estimativa da ETo na região de Araraquara, SP, concluíram que o de Makkink subestimava os valores, em comparação ao método padrão (PMF), o que consiste em um comportamento oposto ao descrito na Tabela 2.

Quanto aos espalhamentos (RMSE), que indicam a faixa de variação da variável dependente para um mesmo valor da variável independente (Carvalho et al., 2015), notou-se que o modelo de Turc apresentou os menores valores (Tabela 3$)$ para 20 EMAs $(71,43 \%)$, inferiores a $0,7688 \mathrm{~mm}$ por dia.

Os modelos de Turc e Radiação Solar apresentaram os melhores ajustes aos dados obtidos com o PMF, de acordo com o índice d de Willmott, com valores de 


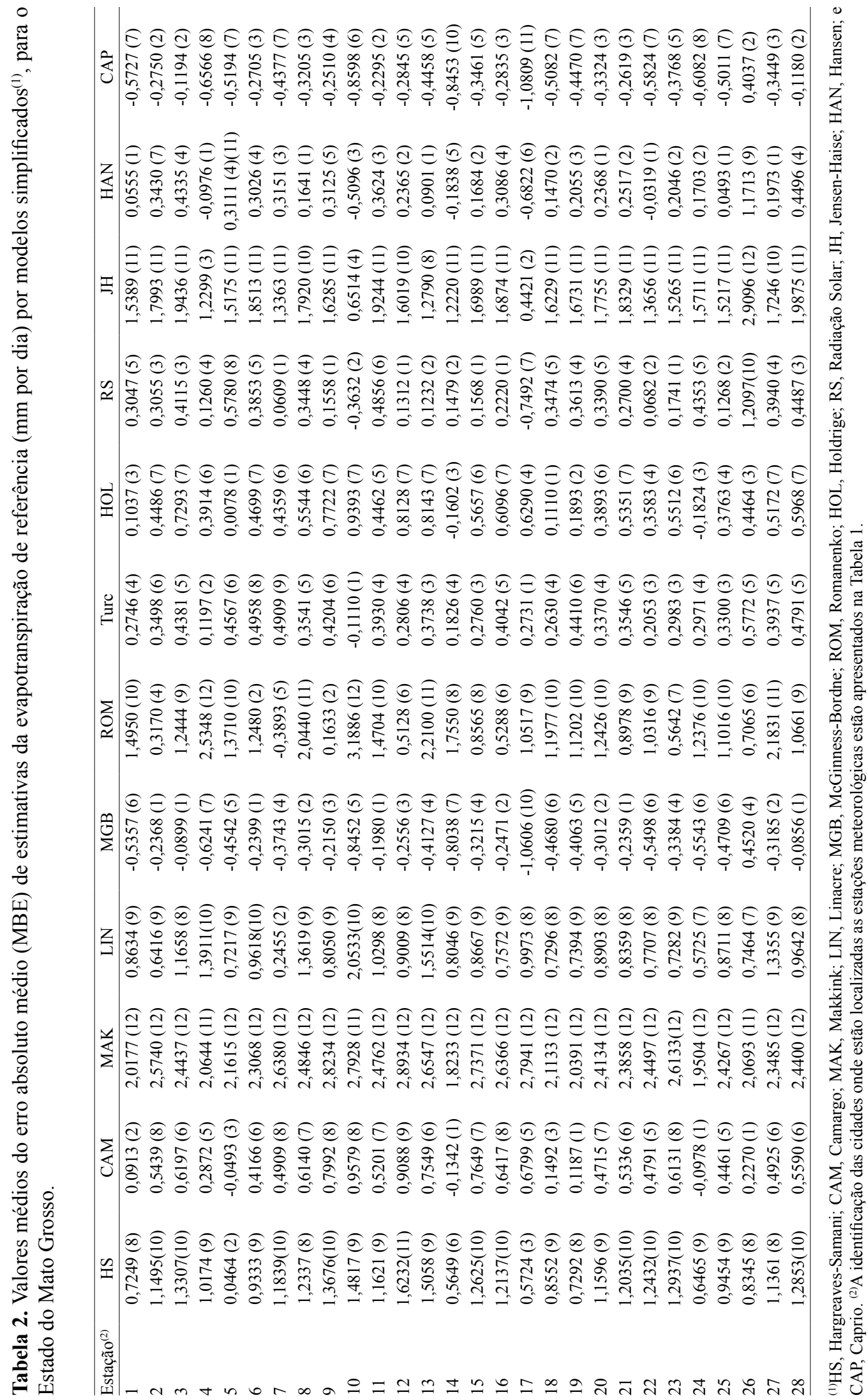


0,8262 a 0,9602 e de 0,7185 a 0,9383 , respectivamente (Tabela 4). Borges \& Mediondo (2007), ao avaliar modelos simplificados de estimativa da ETo, na bacia do Rio Jacupiranga, na região Sudeste do Estado de São Paulo, observaram índices de ajustamento d muito próximos a 1, e recomendaram a utilização da equação de Hargreaves, pela sua simplicidade e confiabilidade.

Contudo, no presentetrabalho, os modelos de Makkink, Romanenko, Jensen-Haise e Hargreaves-Samani apresentaram, em conjunto, os piores indicadores estatísticos na estimativa da ETo, para a escala diária, e, portanto, não se mostraram adequados às condições da região estudada, provavelmente pelo fato de terem sido desenvolvidos em condições climáticas diferentes e para agrupamentos maiores de dados (decendiais ou mensais) (Camargo \& Camargo, 2000). Desse modo, esses modelos podem apresentar melhores resultados com uso de calibrações locais ou regionais dos coeficientes parametrizadas de cada equação (Fernandes et al., 2011; Carvalho et al., 2015).

$\mathrm{Na}$ literatura, há muitas divergências quanto à recomendação de modelos para estimativa da ETo ou de outras variáveis ambientais, em razão da variação observada no desempenho dos modelos com uso de diferentes indicadores estatísticos. Assim, de maneira geral, observa-se que, quando são avaliadas muitas estações meteorológicas, em conjunto com muitos modelos de estimativa, as definições dos melhores desempenhos tornam-se difíceis e, em muitos casos, errôneas. Nesse sentido, a classificação numérica dos modelos de estimativa da ETo, para cada indicador estatístico usado, de acordo com a ordenação de seu desempenho por EMA, pode permitir a definição do melhor modelo para estimativa local da ETo (Tabela 5).

Os modelos de Turc, McGinness-Bordne, Hansen, Radiação Solar e Camargo apresentaram os menores somatórios de $\mathrm{Vp}$ e, consequentemente, a maior frequência de ordenamentos entre os modelos com os melhores desempenhos na estimativa da ETo. Esses modelos apresentaram os melhores desempenhos em 16, 7, 3, 2 e 1 das EMAs avaliadas, respectivamente (Tabela 5). Dessa forma, o uso desses modelos consiste em boa alternativa, em geral, para o dimensionamento e o manejo da irrigação, nas diferentes regiões do Estado do Mato Grosso. A partir desses resultados, entretanto, estabelece-se a necessidade da avaliação da sazonalidade da ETo, em cada EMA, com base no modelo com melhor desempenho.
Na Figura 2, são apresentadas as correlações entre a ETo estimada pelo PMF e a ETo estimada pelos demais modelos para a EMA de Sinop. Constataram-se menores dispersões para os modelos de Camargo, Turc e Caprio, e piores correlações para as estimativas obtidas com os de Jensen-Haise, Romanenko e Makkink. A região de Sinop apresenta médias de precipitações anuais de até 1.974 mm (Souza et al., 2013) e balanço hídrico anual positivo. Conforme Souza et al. (2011), as variações das condições de nebulosidade influenciam o desempenho e o comportamento de modelos de estimativa da ETo. Os autores relatam o exemplo de Seropédica, RJ, onde os modelos Radiação Solar e Camargo apresentaram os melhores resultados para as estimativas em condições de céu aberto (transmissividade atmosférica superior a $65 \%$ ) e de céu nublado (transmissividade atmosférica inferior a $35 \%$ ), respectivamente.

Nesse contexto, segundo Conceição (2003), os métodos que empregam somente a temperatura do ar, como é o caso dos modelos de Linacre, Romanenko e Holdridge, permitem uma representatividade limitada, uma vez que, para uma mesma temperatura, podem ocorrer valores diferentes de umidade do ar, o que indica que a demanda hídrica pode variar sem que haja, necessariamente, alteração da temperatura do ar.

As pesquisas com estimativas da ETo traduzem variação no comportamento dos modelos, nas diferentes regiões brasileiras, como relatado por diversos autores (Borges \& Mediondo, 2007; Oliveira et al., 2008; Syperreck et al., 2008; Oliveira et al., 2010; Tagliaferre et al., 2010; Alencar et al., 2011; Fernandes et al., 2011; Melo \& Fernandes, 2012; Souza et al., 2011). Dessa forma, a seleção do método a ser empregado depende de fatores como condições climáticas, disponibilidade de dados meteorológicos, complexidade do método, agrupamento dos dados considerados e custos (Almeida et al., 2010; Souza et al., 2011). Em muitos casos, modelos que não apresentam desempenhos satisfatórios para estimativas em uma região devem ser avaliados e, se necessário, calibrados para as condições climáticas locais ou regionais, como realizado no presente estudo e nos trabalhos de Todorovic et al. (2013), com estimativas em 577 estações meteorológicas, distribuídas em 16 países do Mediterrâneo, e de Carvalho et al. (2015), com avaliações em 44 estações meteorológicas convencionais na região Sudeste do Brasil. 


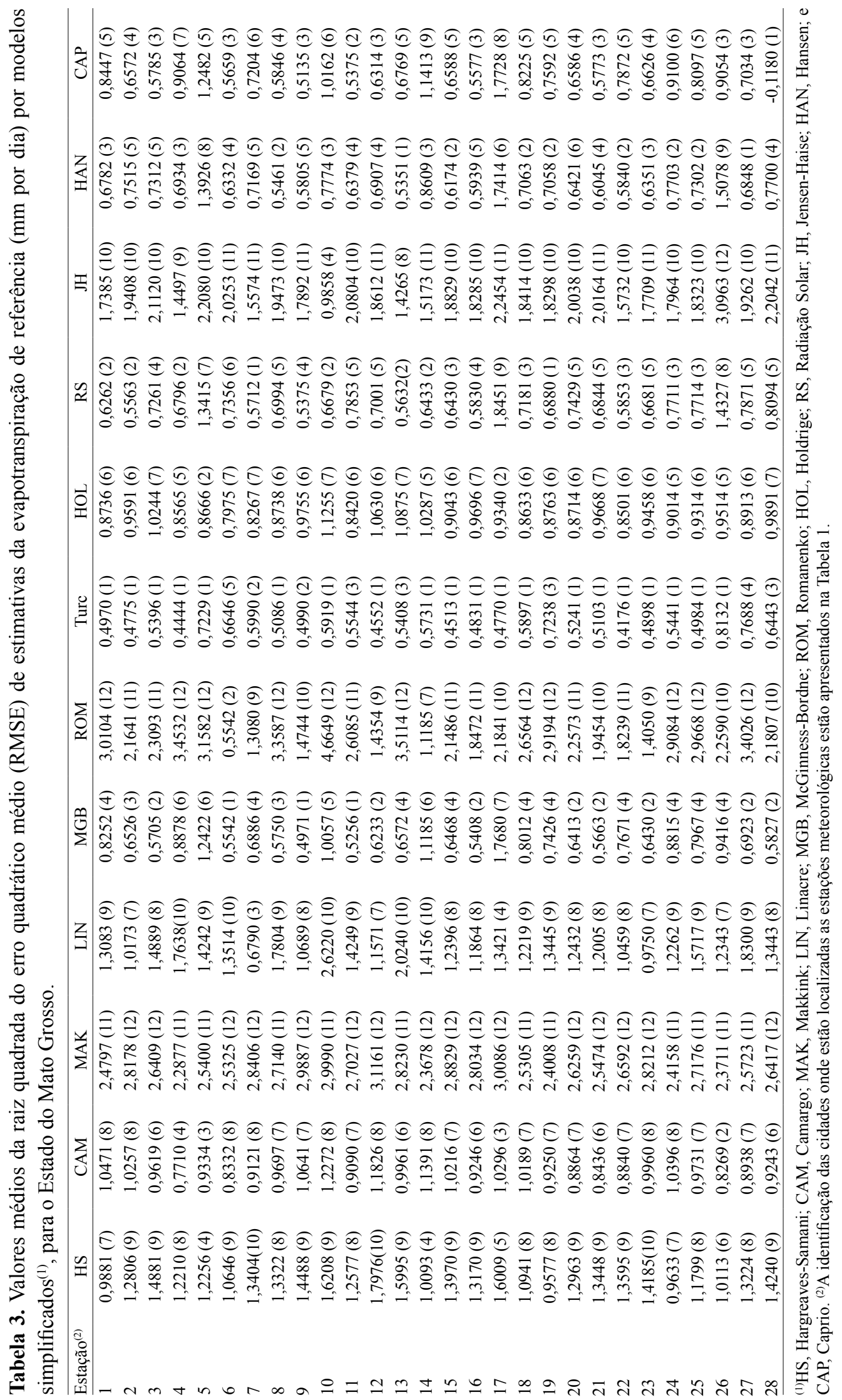




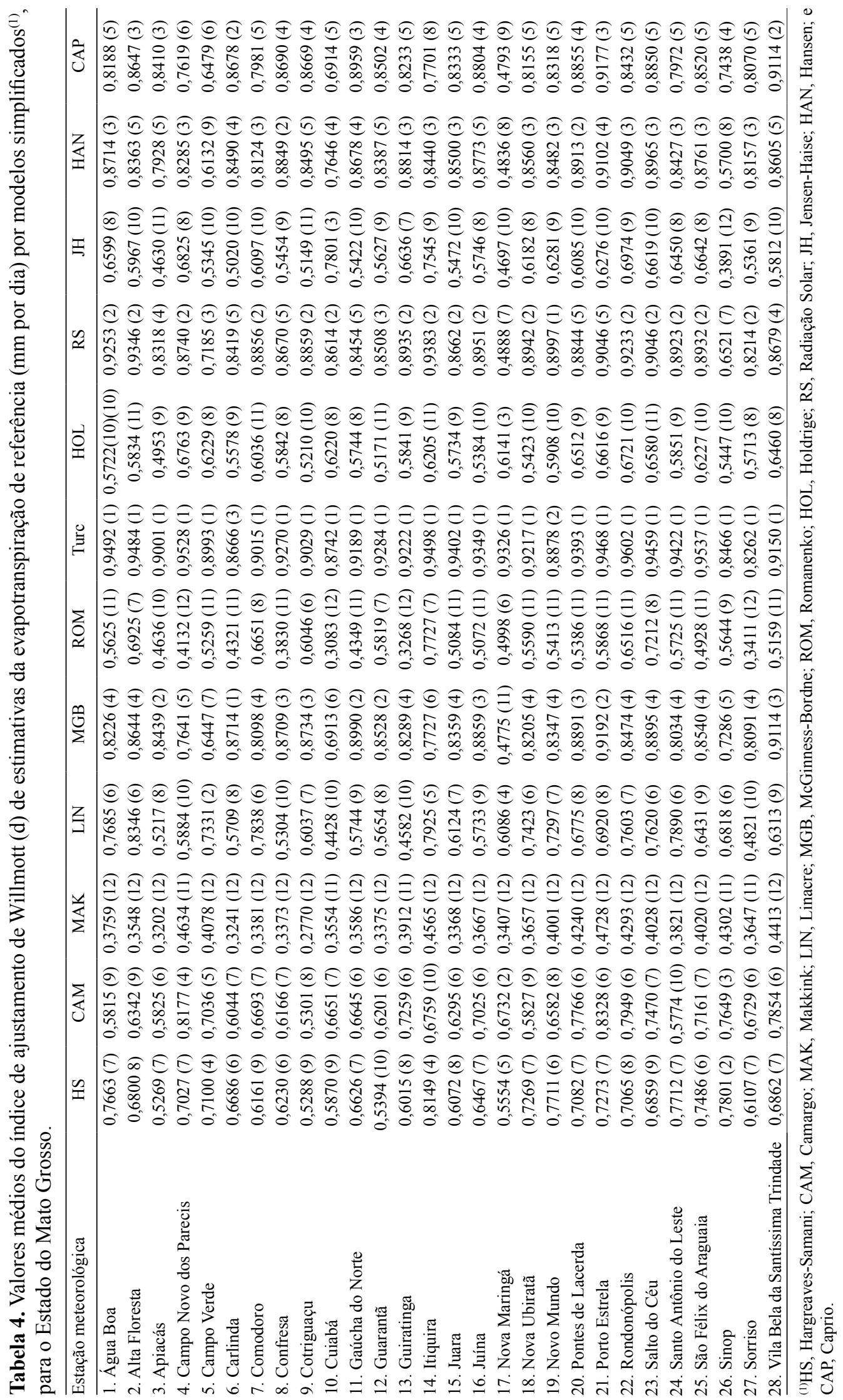




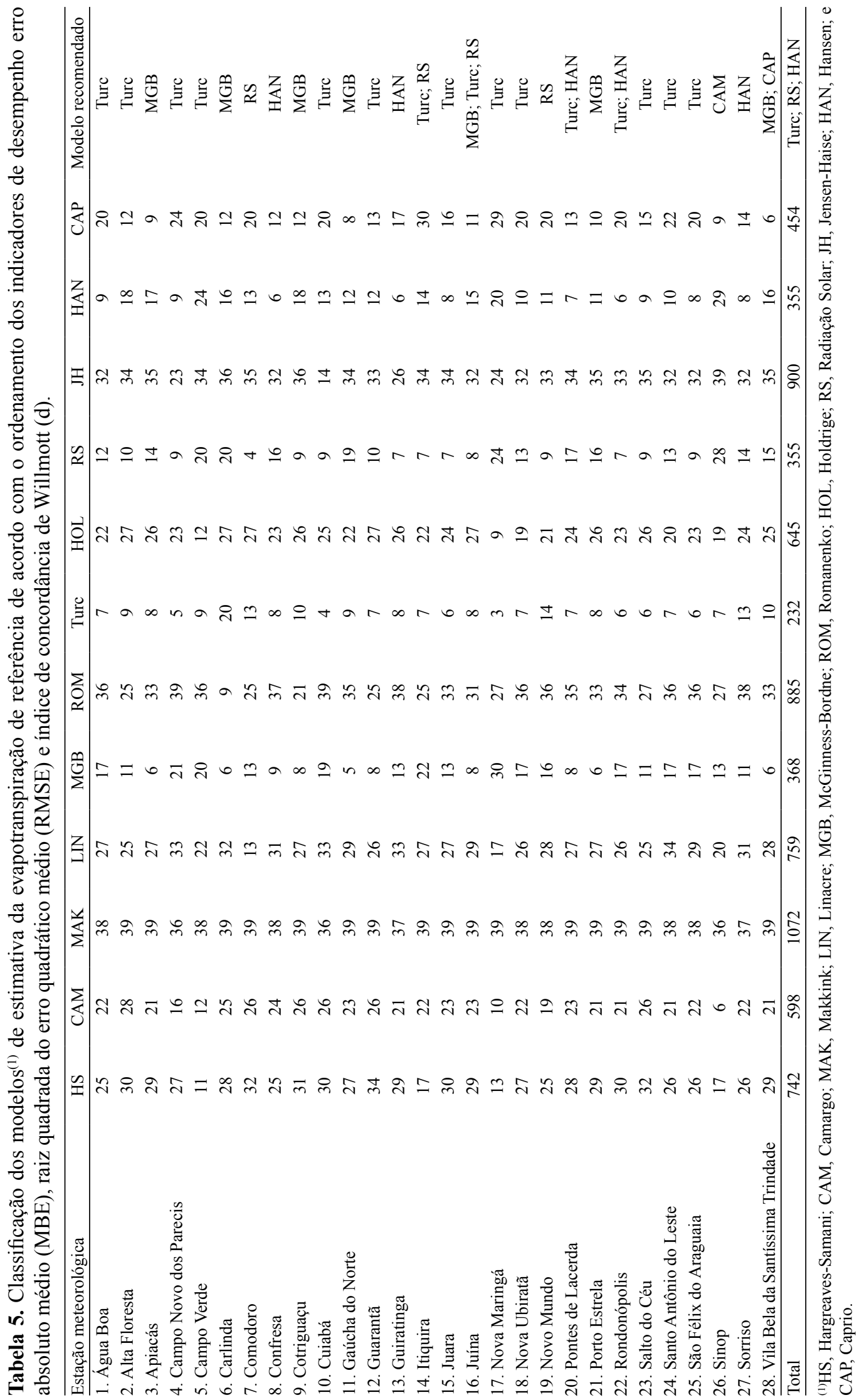



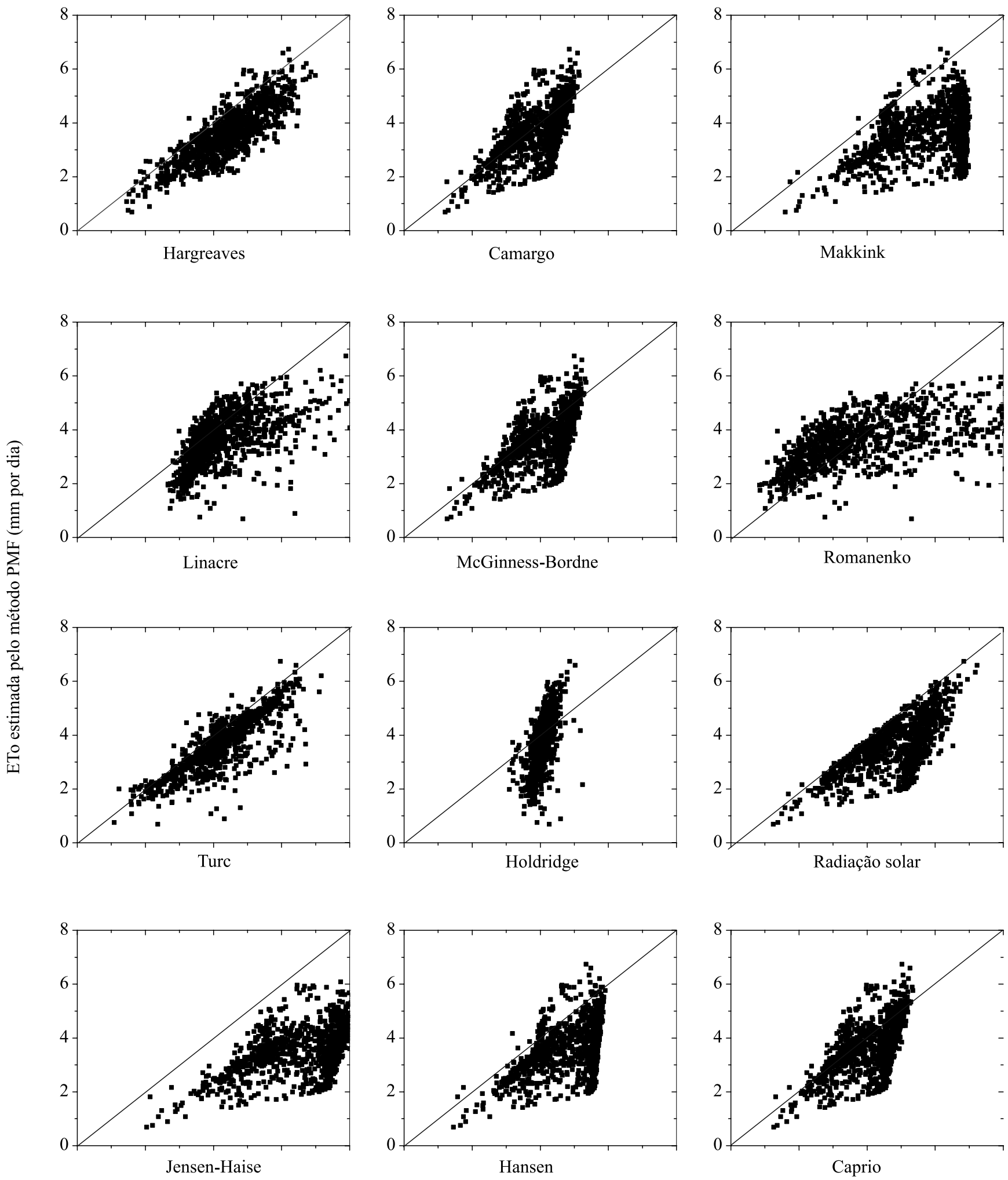

ETo estimada pelos modelos estudados (mm por dia)

Figura 2. Correlações entre a evapotranspiração de referência (ETo) estimada pelo método de Penman-Monteith FAO 56 (PMF) e pelos modelos simplificados avaliados, para a estação meteorológica automática de Sinop, MT. 


\section{Conclusões}

1. Quando apenas dados de temperatura do ar e umidade relativa do ar estão disponíveis, os modelos simplificados de estimativa da evapotranspiração de referência diária recomendados para o Estado do Mato Grosso são os de Turc, McGinness-Bordne, Radiação Solar, Hansen e Camargo.

2. Os modelos de Romanenko, Makkink e Holdridge não são recomendados para o Estado do Mato Grosso e necessitam de calibração regional dos seus coeficientes.

\section{Agradecimentos}

Ao Instituto Nacional de Meteorologia (Inmet), pela disponibilização dos dados; e ao Conselho Nacional de Desenvolvimento Científico e Tecnológico (CNPq, processo 142210/2009-9), pela concessão de bolsa.

\section{Referências}

ALENCAR, L.P. de; SEDIYAMA, G.C.; WANDERLEY, H.S.; ALMEIDA, T.S.; DELGADO, R.C. Avaliação de métodos de estimativa da evapotranspiração de referência para três localidades no Norte de Minas Gerais. Revista Engenharia na Agricultura, v.19, p.437-449, 2011. DOI: 10.13083/1414-3984.v19n05a05.

ALlEN, R.G.; PEREIRA, L.S.; RAES, D.; SMITH, M. Crop evapotranspiration: guidelines for computing crop water requirements. Rome: FAO, 1998. 297p. (FAO Irrigation and Drainage Paper, 56).

ALMEIDA, B.M. de; ARAÚJO, E.M. de; CAVALCANTE JUNIOR, E.G.; OLIVEIRA, J.B. de. Comparação de métodos de estimativa da ETo na escala mensal em Fortaleza-CE. Revista Brasileira de Agricultura Irrigada, v.4, p.93-98, 2010. DOI: 10.7127/RBAI.V4N200610.

ALVES SOBRINHO, T.; RODRIGUES, D.B.B.; OLIVEIRA, P.T.S. de; REBUCCI, L.C.S.; PERTUSSATTI, C.A. Estimativa da evapotranspiração de referência através de redes neurais artificiais. Revista Brasileira de Meteorologia, v.26, p.197-203, 2011. DOI: 10.1590/S0102-77862011000200004.

BEZERRA, J.R.C.; AZEVEDO, P.V. de; SILVA, B.B. da; DIAS, J.M. Evapotranspiração e coeficiente de cultivo do algodoeiro BRS-200 Marrom, irrigado. Revista Brasileira de Engenharia Agrícola e Ambiental, v.14, p.625-632, 2010. DOI: 10.1590/ S1415-43662010000600009.

BORGES, A.C.; MENDIONDO, E.M. Comparação entre equações empíricas para estimativa da evapotranspiração de referência na Bacia do Rio Jacupiranga. Revista Brasileira de Engenharia Agrícola e Ambiental, v.11, p.293-300, 2007. DOI: 10.1590/ S1415-43662007000300008.

BRISTOW, K.L.; CAMPBELL, G.S. On the relationship between incoming solar radiation and daily maximum and minimum temperature. Agricultural and Forest Meteorology, v.31, p.159-166, 1984. DOI: 10.1016/0168-1923(84)90017-0.

CAMARGO, A.P. de; CAMARGO, M.B.P. de. Uma revisão analítica da evapotranspiração potencial. Bragantia, v.59, p.125-137, 2000.

CAPRIO, J.M. The solar thermal unit concept in problems related to plant development and potential evapotranspiration. In: LIETH, H. (Ed.). Phenology and seasonality modeling. Berlin: Springer Verlag, 1974. p.353-364. (Ecological studies, 8). DOI: 10.1007/978-3-642-51863-8_29.

CARVALHO, D.F. de; ROCHA, H.S. da; BONOMO, R.; SOUZA, A.P. de. Estimativa da evapotranspiração de referência a partir de dados meteorológicos limitados. Pesquisa Agropecuária Brasileira, v.50, p.1-11, 2015. DOI: 10.1590/ S0100-204X2015000100001.

CARVALHO, L.G. de; RIOS, G.F.A.; MIRANDA, W.L.; CASTRO NETO, P. Evapotranspiração de referência: uma abordagem atual de diferentes métodos de estimativa. Pesquisa Agropecuária Tropical, v.41, p.456-465, 2011. DOI: 10.5216/pat.v41i3.12760.

CONCEIÇÃO, M.A.F. Estimativa da evapotranspiração de referência com base na temperatura do ar para as condições do Baixo Rio Grande, SP. Revista Brasileira de Agrometeorologia, v.11, p.229-236, 2003.

CONCEIÇÃO, M.A.F.; MANDELLI, F. Comparação entre métodos de estimativa da evapotranspiração de referência em Bento Gonçalves, RS. Revista Brasileira de Agrometeorologia, v.13, p.303-307, 2005.

DOORENBOS, J.; KASSAN, A.H. Efeito da água no rendimento das culturas. Tradução de H. R. Gheyi; A. A. de Sousa; F. A. V. Damasceno; J. F. de Medeiros. Campina Grande: UFPB, 1994. 306 p. (FAO. Estudos FAO. Irrigação e drenagem, 33).

FERNANDES, A.L.T.; FRAGA JÚNIOR, E.F.; TAKAY, B.Y. Avaliação do método Penman-Piche para a estimativa da evapotranspiração de referência em Uberaba, MG. Revista Brasileira de Engenharia Agrícola e Ambiental, v.15, p.270-276, 2011. DOI: 10.1590/S1415-43662011000300008.

HANSEN, S. Estimation of potential and actual evapotranspiration. Hydrology Research, v.15, p.205-212, 1984.

HENRIQUE, F. de A.N.; DANTAS, R.T. Estimativa da evapotranspiração de referência em Campina Grande, Paraíba. Revista Brasileira de Engenharia Agrícola e Ambiental, v.11, p.594-599, 2007. DOI: 10.1590/S1415-43662007000600007.

HOLDRIDGE, L.R. Simple method for determining potential evapotranspiration from temperature data. Science, v.130, p.572, 1959. DOI: $10.1126 /$ science. 130.3375 .572 .

MELO, G.L. de; FERNANDES, A.L.T. Evaluation of empirical methods to estimate reference evapotranspiration in Uberaba, state of Minas Gerais, Brazil. Engenharia Agrícola, v.32, p.875-888, 2012. DOI: 10.1590/S0100-69162012000500007.

MONTEIRO, E.B.; SILVA, C.C. da; SILVA, A.C. da; SOUZA, A.P. de. Estimating emission of leaves seedlings forest in different shading levels, at conditions of Transition Amazon-Cerrado, Brazil. American Journal of Plant Sciences, v.5, p.2330-2341, 2014. DOI: 10.4236/ajps.2014.515247. 
OLIVEIRA, G.M. de; LEITÃO, M. de M.V.B.R.; BISPO, R. de C.; SANTOS, I.M.S.; ALMEIDA, A.C. de. Comparação entre métodos de estimativa da evapotranspiração de referência na região Norte da Bahia. Revista Brasileira de Agricultura Irrigada, v.4, p.104-109, 2010. DOI: 10.7127/rbai.v4n206100.

OLIVEIRA, L.M.M. de; MONTENEGRO, S.M.G.L.; AZEVEDO, J.R.G. de; SANTOS, F.X. dos. Evapotranspiração de referência na bacia experimental do riacho Gameleira, PE, utilizando-se lisímetro e métodos indiretos. Revista Brasileira de Ciências Agrárias, v.3, p.58-67, 2008. DOI: 10.5039/agraria.v3i1a250.

OUDIN, L.; HERVIEU, F.; MICHEL, C.; PERRIN, C.; ANDRÉASSIAN, V.; ANCTIL, F.; LOUMAGNE, C. Which potential evapotranspiration input for a lumped rainfall-runoff model?: Part 2 - towards a simple and efficient potential evapotranspiration model for rainfall-runoff modeling. Journal of Hydrology, v.303, p.290-306, 2005. DOI: 10.1016/j. jhydrol.2004.08.026.

PEREIRA, A.R.; VILLA NOVA, N.A.; SEDIYAMA, G.C. Evapotranspiração. Piracicaba: FEALQ, 1997. 183 p.

RAZIEI, T.; PEREIRA, L.S. Estimation of ETo with Hargreaves-Samani and FAO-PM temperature methods for a wide range of climates in Iran. Agricultural Water Management, v.121, p.1-18, 2013. DOI: 10.1016/j.agwat.2012.12.019.

SOUZA, A.P. de; CARVALHO, D.F. de; SILVA, L.B.D. da; ALMEIDA,F.T.de; ROCHA,H.S. Estimativas da evapotranspiração de referência em diferentes condições de nebulosidade. Pesquisa Agropecuária Brasileira, v.46, p.219-228, 2011. DOI: 10.1590/ S0100-204X2015000100001.

SOUZA, A.P. de; MOTA, L.L. da; ZAMADEI, T.; MARTIM, C.C.; ALMEIDA, F.T. de; PAULINO, J. Classificação climática e balanço hídrico climatológico no estado de Mato Grosso. Nativa, v.1, p.34-43, 2013. DOI: 10.14583/2318-7670.v01n01a07.
STONE, R. J. Improved statistical procedure for the evaluation of solar radiation estimation models. Solar energy, v.51, p.289- 291, 1993. DOI: 10.1016/0038-092X(93)90124-7.

SYPERRECK, V.L.G.; KLOSOWSKI, E.S.; GRECO, M.; FURLANETTO, C. Avaliação de desempenho de métodos para estimativas de evapotranspiração de referência para a região de Palotina, Estado do Paraná. Acta Scientiarum. Agronomy, v.30, p.603-609, 2008. Suplemento.

TAGLIAFERRE, C.; SILVA, R.A. de J.; ROCHA, F.A.; SANTOS, L. da C.; SILVA, C. dos S. da. Estudo comparativo de diferentes metodologias para determinação da evapotranspiração de referência em Eunápolis-BA. Revista Caatinga, v.23, p.103-111, 2010.

TANAKA, A.A. Avaliação de métodos de estimativa da radiação solar global e da evapotranspiração de referência para o estado de Mato Grosso. 2013. 110p. Tese (Doutorado) - Universidade Estadual Paulista, Botucatu.

TODOROVIC, M.; KARIC, B.; PEREIRA, L.S. Reference evapotranspiration estimate with limited weather data across a range of Mediterranean climates. Journal of Hydrology, v.481, p.166-176, 2013. DOI: 10.1016/j.jhydrol.2012.12.034.

VESCOVE, H.V.; TURCO, J.E.P. Comparação de três métodos de estimativa da evapotranspiração de referência para a região de Araraquara - SP. Engenharia Agrícola, v.25, p.713-721, 2005. DOI: $10.1590 / \mathrm{S} 0100-69162005000300017$.

VISWANADHAM, Y.; SILVA FILHO, V.P.; ANDRÉ, R.G.B. The Priestley-Taylor parameter $\alpha$ for the Amazon forest. Forest Ecology and Management, v.38, p.211-225, 1991. DOI: 10.1016/0378-1127(91)90143-J.

WILLMOTT, C.J.; ACKLESON, S.G.; DAVIS, R.E.; FEDDEMA, J.J.; KLINK, K.M.; LEGATES, D.R.; O’DONNELL, J.; ROWE, C.M. Statistics for the evaluation and comparison of models. Journal of Geophysical Research, v.90, p.8995-9005, 1985. DOI: 10.1029/JC090iC05p08995.

Recebido em 8 de maio de 2015 e aprovado em 22 de dezembro de 2015

Pesq. agropec. bras., Brasília, v.51, n.2, p.91-104, fev. 2016

DOI: 10.1590/S0100-204X2016000200001 